\title{
Evaluation of bone excision effects on a human skull model - I: mechanical testing and digital image correlation
}

\section{Constantinos Franceskides ${ }^{1}$, Thibault Leger $^{2}$, Ian Horsfall ${ }^{3}$, Gianluca Tozzi ${ }^{4}$, Michael Gibson ${ }^{5}$, Peter Zioupos ${ }^{* 1}$}

${ }^{1}$ Musculoskeletal and Medicolegal Research Group, Cranfield Forensic Institute, Cranfield University, Defence Academy of the UK, Shrivenham, SN6 8LA, UK

${ }^{2}$ Materials Science, University of Paris-Sud, Orsay, France

${ }^{3}$ Centre for Defence Engineering, Cranfield University, Defence Academy of the UK, Shrivenham, SN6 8LA, UK

${ }^{4}$ School of Engineering, University of Portsmouth, Anglesea Road, Portsmouth, PO1 3DJ, UK

${ }^{5}$ Centre for Simulation \& Analytics, Cranfield University, Defence Academy of the UK; Shrivenham, SN6 8LA, UK

\begin{abstract}
The mechanisms of skull impact loading may change following surgical interventions such as the removal of bone lesions, but little is known about the consequences in the event of subsequent head trauma. We therefore prepared acrylonitrile butadiene styrene human skull models based on clinical computed tomography skull data using a 3D printer. Six replicate physical skull models were tested, three with bone excisions and three without. A drop-tower was used to simulate the impact sustained by falling backwards onto the occipital lobe region. The impacts were recorded with a high-speed camera and the occipital strain response was determined by digital image correlation (DIC). Although the hole affected neither the magnitude nor the sequence of the fracture pattern, DIC analysis highlighted an increase in strain around the excised area (0.45$16.4 \%$ of the principal strain). Our approach provides a novel method that could improve the quality of life for patients on many fronts, including protection against trauma, surgical advice, post-operative care, advice in litigation cases, as well as facilitating general biomechanical research in the area of trauma injuries.
\end{abstract}

\section{Keywords}

Biopsy; Bone fracture; Strains; Digital Image Correlation; Physical models; Impact 


\section{Introduction}

Between 2010 and 2015, more than 1 million falls resulting in head injuries, averaging 200,000 per year, were recorded by the Health and Social Care Information Centre (HSCIC) of the United Kingdom. ${ }^{1}$ During the same period, an average of 441 skull biopsies were conducted per year (Table 1). The consequences of bone and soft tissue tumour surgery, whether for biopsy or full excision, have been investigated since the 1970 s. $^{2}$ However, most of these investigations have focused on the biological (histological) impact, with only a few considering the biomechanical aspects. ${ }^{3,4}$ Therefore, it is unclear how the mechanisms of head impact loading may change following surgical interventions such as the removal of bone lesions. The probability, however small, of having a head impact after a skull biopsy is not negligible considering the statistics mentioned above.

With technological advances in biomedical and biomechanical modelling, many researchers have attempted to understand ${ }^{5}$ or even predict ${ }^{6}$ musculoskeletal behaviour under different loading conditions. With this in mind, the prediction of bone excision effects could be improved by using previous biomechanical research in concert with evolving technological and mechanical testing methods. The main problems that must be addressed when examining a real-life/real-time loading scenario revolve around three aspects, namely: (1) representative biofidelic loading conditions (in terms of time and boundary effects), (2) accurate, real-time (fast), reliable and non-contact recording of the deformations, (3) reproducibility of the tests and the samples in order to minimize and control for unknown factors such as biological variability (no real-life sample is like any other, each biological sample is unique), and (4) ultimately the material properties of the biological sample. The present study addresses these challenges by combining three modern techniques: drop tower impact loading, digital image correlation (DIC) and 3D rapid prototype printing following computed tomography (CT) scanning.

DIC is a non-contact data acquisition method which achieves full-field strain mapping through the analysis of consecutive images captured by camera. The speed and specifications of the camera allow the rapid capture of high-quality images to facilitate strain analysis in real-time and in high-speed scenarios. DIC has been combined with impact loading of the musculoskeletal system to evaluate effects such as ballistic helmet trauma in the military environment ${ }^{7}$ or to develop and validate a model of infant skull impact loading. ${ }^{8}$ Furthermore, patient-specific models have been used to validate the conformity of implants by combining finite element analysis (FEA) with DIC. ${ }^{9,10}$ Drop towers are modern instrumented versions of dead-weight impact loading systems, allowing accelerated loading with precise determination of the energy input. Threedimensional physical model prototype printing has evolved into the most powerful method for the production of life-like models of natural structures. ${ }^{9,11}$ Depending on the sophistication, 3D printing could replicate the fine structural details of bones by using ever smaller nozzle sizes and different grade of polymers. In latest printer models a range of up to 12 polymers of different properties can be deposited by one and the same printer in one run producing a mosaic of interlaced microvolumes thus mimicking the inner microstructure of natural materials. The detailed information that drives this intrinsic 3D printing is taken from modern CT scans, which can divide structures into voxels of just a few micrometres. ${ }^{11}$ 
The possibilities that are open to a biomechanician to work on certain bespoke studies are relatively limited: cadaveric tests, computational studies and we suggest physical model analogues. The latter offer hands on experience and cost-effective insight into the deformation and fracture process. By a combination of the modern powerful methods mentioned above one may be allowed to analyse the loading of mechanically compromised skulls in real time. And despite the advances described above, previous studies have not addressed the influence of biopsies and excisions on the structural integrity of a human skull when exposed to impact loading. We therefore used a 3D printer to prepare human skull models based on clinical CT data from a case study, and then compared the mechanical behaviour of skulls with and without bone excisions in a simulated fall, causing an impact on the occipital lobe region. Our hypothesis was that skull surgery would have a demonstrable effect on the deformation behaviour, the dissipation of loading forces and possibly on the crack growth patterns too.

\section{Materials and methods}

\section{The patient}

The replica skulls used in this study were modelled on a 28 -year-old male patient who had no previous history of musculoskeletal disorders and no prior bone tissue surgery. The excision to the occipital skull region was performed by a consultant neurosurgeon at Wellington Hospital, London, UK, in the context of investigating the presence of a focal point of bone malignancy. The patient was otherwise healthy, but concerned that the excision would have compromised the structural strength of his skull. Ethical approval for the work was granted by the relevant Cranfield University committee and the patient consented under the approval reference CURES/787/2016.

\section{Creation of physical skull models}

Six physical skull models were prepared, based on the same post-operative clinical CT scan acquired at a resolution of $0.488 \times 0.488 \times 1.2 \mathrm{~mm}$ and at $120 \mathrm{kV}$ and $400 \mathrm{~mA}$. Two distinct skull geometries were created (with and without the excision) using Simpleware ScanIP v7 (https://www.simpleware.com/software/scanip/) (Figure 1). The data were imported as a 32-bit float, but were converted to an 8-bit float and resampled (using the cubic function) to match the voxel axial dimension to the in-plane dimension with the resulting cubic voxels of side length $0.488 \mathrm{~mm}$. A series of masks was then applied, with the appropriate grayscale thresholding and flood fill operations to select only bone matter within the scanned volume. A Gaussian smoothing filter was used to better represent the skull geometry and negate as much as possible to the stepped appearance of voxel-based output from CT scanners.

The normal skull geometry was created using the neighbouring morphology, such as the surrounding bone thickness and curvature, thus mimicking as closely as possible the preoperative condition of the region. The three skull models with an excision (S1, S2 and S3) and the three without (S4, S5 and S6) were imported to Stratasys CatalystEX to create the tool-path files, and then uploaded into the printer. The six physical skull models were printed on an FDM Stratasys uPrint SE 3D printer 
(http://www.stratasys.com/3d-printers) fitted with a 0.4-mm nozzle, using the acrylonitrile butadiene styrene (ABS) material supplied by the manufacturer. The support fill was set to 'smart' and the model interior to 'solid'. This resulted in a typical printing time of $42 \mathrm{~h}$ per skull, requiring almost a complete reel of material (33 cubic inches). ABS was chosen as being the most common choice of material for many users, so that the results are of wide interest for the biomechanics community. The precise choice of settings resulted from other tests we have conducted on material samples with simple and complex geometries, where we have demonstrated that the force/displacement curves of real biological and 3D-printed ABS samples were identical in the elastic region ${ }^{12}$. This validation study ${ }^{12}$ showed that, while there is very good agreement between the ABS and bone in the elastic region, there is very poor agreement in the plastic region where fracture occurs. This is inevitable as bone is a semi-brittle solid with a very short postyield strains and a slightly lower yield stress. ABS by contrast is an elastomer with ability to draw to larger strains and exhibiting no brittle behaviour. It should be made clear that use of ABS therefore, is being made in the paper in this context to enlighten us on the pre-yield effects and phenomena.

\section{Refinement of the model skulls}

Perma-Gel was used to fill the models in order to mimic the properties of the brain and reproduce its energy absorption and response. ${ }^{13}$ The total mass of each model was $\sim 2.1$ $\mathrm{kg}$ with a maximum variance of $0.040 \mathrm{~kg}$. A solid base was created, also from PermaGel, to allow the repositioning of the skulls, thus eliminating orientation errors. All samples were prepared in a similar manner as previously reported ${ }^{15-16}$ with a randomized speckle pattern. The black-to-white ratio was kept as close as possible to 1:1, and Castrol White Contrast Paint 710 (2386) non-strippable aerosol was used to apply the highcontrast background evenly over the model surface. Finally, the size of the speckles (Figure 2) was determined using the following equations: ${ }^{10,17}$

$$
\begin{gathered}
S_{\text {ideal }}=\frac{4 \pm 1}{M} \\
M_{\text {factor }}=\frac{n_{\text {length sensor pixels }}}{d_{\text {length }}}
\end{gathered}
$$

\section{Drop tests}

The drop tests were conducted using an Imatek IM10 drop tower (http://www.imatek.co.uk/) with a total carriage and striker mass of $2.91 \mathrm{~kg}$ (Figure 3). At the end of the carriage, a 45-mm circular titanium striker was fitted with a dampening surface which was adapted from a Humanetics Hybrid III headform thickness of $12.7 \mathrm{~mm}$ (http://www.humaneticsatd.com/) with $43 \pm 5$ shore hardness ' $A$ '. This provided a dampening effect and hence a smoother ramp rate, simulating the soft tissues surrounding a real human skull. ${ }^{18,19}$ The rubber was manufactured to the specifications described above by Watts Urethane Products Ltd (https://wattspolyurethane.co.uk/). The skulls were subjected to a load on the occipital region proximal to the excision site, mimicking 
the effect of an unconscious or incapacitated human falling backwards and striking this region on a hard surface.

DIC data were acquired via a series of images captured by two V12.1 Phantom highspeed cameras that were set at a stereo incident angle of $25^{\circ}$, recording at $15,000 \mathrm{fps}$. The camera was commanded using PCC standalone software (https://www.highspeedcameras.com/Service-Support/PCC-Software) which provided a falling edge signal input from the drop tower to ensure synchronous capture. The data were analysed using Aramis GOM software (http://www.gom.com/metrologysystems/system-overview/aramis.html) with quadrangle facets at 19 pixels (size) and 17 pixels (step), 484 3D points generated over 176 stages, and an average intersection deviation of $0.0150 \mathrm{~mm}$.

Due to the confined space of the drop-tower base, artificial lighting was installed to provide consistent lighting conditions. This was achieved using three light sources, namely two conventional halogen lights with focal adjustment, and a light emitting diode (LED) Cree flood light acting as a background source, which was reflected onto the object using a flat mirror placed outside the impact area.

Two series of tests were conducted, with varying striker velocities of $\sim 8 \mathrm{~m} / \mathrm{s}$ at $100 \mathrm{~J}$ (T1) and $\sim 9.75 \mathrm{~m} / \mathrm{s}$ at $130 \mathrm{~J}$ (T2). These speeds are for the striker not the speeds by which the skull would impact the ground in free fall. The striker speeds were chosen to match biomimetically the energies in a free fall case. The lower-energy impacts (T1) were used to determine the loading response under the defined testing analogy $\left(50^{\text {th }}\right.$ percentile male adult losing consciousness and falling with direct impact on the occipital region), and the higher-energy impacts (T2) were used to induce failure in the skulls and thus determine the fracture pattern and propagation path. We have to add that a series of preliminary tests were needed, and were conducted to get a feel of what speeds (or better say input energies will create what effects). The two input energies used showed for the physical model of the skull: no damage for 100J and damage been present at 130J. It is remarkable that these energy values and the resultant effects are similar to skull values dropping from similar height. in vivo. Although one will argue that ABS skull is not a 'real' skull one has to agree that even in this respect the physical plastic skulls behave like real ones.

\section{Preliminary tests on ABS cubes}

Before producing the physical skull models, preliminary tests were conducted on four 3D-printed ABS cubes with dimensions of $40 \times 40 \times 40 \mathrm{~mm}$ (Figure 4) to address some inconsistency in the literature concerning the anisotropy of 3D-printed objects. Some reports indicate a difference in compressive performance depending on the orientation of layers ${ }^{20}$ whereas others do not ${ }^{21}$ thus making it necessary to investigate the variation of layer deposition for this specific printer and material. The dark gray surface in Figure 4 indicates where the load was applied, and the line represents the orientation of ABS layer deposition. The cubes were compressively loaded between platens, until failure, using a Zwick Roell 1484 fitted with a $200 \mathrm{kN}$ load cell (www.zwick.co.uk) at rate of $0.5 \mathrm{~mm} / \mathrm{min}$. The results (Table 2) indicated that, despite some layer directionality and inter-laminar bonding, the difference in stiffness was less than $16 \%$ (max vs min values) indicating that the $3 \mathrm{D}$ printed structures were practically isotropic. 


\section{Results}

\section{Tests on the skull models}

Eight tests were conducted, six in the T1 state and two in the T2 state. During the T1 test series, model S2 suffered a premature failure, which resulted in a greater shear angle in the proximal region of the excision leading to a lower strain recording (Figures 5 and 6). Admittingly the behaviour in the elastic region is very important and perhaps it can be more easily replicated in 3D models. The behaviour in the plastic region is more difficult to make it work out. Hence, we only loaded to destruction (cracking) 2 out of the 6 skulls and kept 4 to use again in further studies of loading and with other boundary conditions. It was prudent and economical to do so. Figure 7 shows models S3 and S4 with the cracks numbered in order of appearance and a rough trace of the extent of each crack. During the T2 test series, models S3 (with a hole) and S4 (without a hole) both failed, showing three distinct crack propagation paths. In both cases, the cracks converged to the point of impact from 3 directions. Crack propagation was very similar in both cases, in extent and orientation. The high-speed videography clearly showed that even the crack branching sequence was the same, not only the manner and sequence of crack propagation, but also the extent.

DIC analysis clearly revealed strain concentration on the perimeter of the excision, but not in the case of the healthy skull (Figure 8). This compressive strain concentration was more noticeable while the skulls were in the elastic loading phase of the tests (all of the $\mathrm{T} 1$ and prior to the cracking phase in $\mathrm{T} 2$ tests) of the samples. The maximum principal strains were in the vicinity of the hole. The drop-tower data also revealed consistent velocity decay in each of the skulls (Figure 9). The graduation tendency was similar in the T1 and T2 test environments, with only the magnitude (representing impact speed) differing between the test series. The force decay was also similar in the T1 and T2 test series (Figure 10) with the only major difference being the magnitude of the force, even though the T2 test series induced failure in the models. Figure 11 shows the test screen in Aramis GOM software which displayed synchronous behaviours of load, strain mapping, video play and range of strain all evident in one screen frame. The synchronicity of all these signals allows interpretations to be made relatively easily as to the occurrence of events, they sequence and how they are related to each other.

\section{Discussion}

Little is known about the biomechanical effect of surgical procedures such as bone lesion removal on the response of human skulls to impact trauma. Such interventions may have a detrimental effect on the load response of natural musculoskeletal structures because the integrity of the structure is compromised. This gives rise to legitimate concerns about the morbidity and mortality that may ensue in the aftermath of such procedures if the skull is exposed to subsequent impact trauma.

We prepared physical skull models from a patient with a surgical hole in the occipital region and similar skull models with the excision hole filled to represent the preoperative state. Three skull models in each configuration were then subjected to drop- 
tower tests with two impact velocities in order to test the strain responses (T1, low impact) and failure characteristics (T2, high impact). The test set up was not natural because it involves a striker hitting a skull which was securely held on a soft substrate. In real world scenarios it is like a free-falling skull hitting for instance a hard surface. However, using in-lab equipment and the instrumented drop tower allows a degree of control over a number of other confounding test variables. Being able to record and impact the same area was more important and prominent in understanding the load dissipation proximal to the excision. Eventually all our tests were carried out in a single day to minimize errors and limit the number of models required for testing over the range of velocities/energies we considered.

Within the first group model S2 showed a lower principal strain value than models S1 and S3 and experienced failure under milder test conditions. This probably reflected an unanticipated printing anomaly caused by a temporary nozzle blockage and the consequential pause in printing, which allowed the already layered material to solidify, creating a weaker bond. Less energy was therefore absorbed (compared to the other models) before failure, and the resultant energy translated to less elastic deformation. S2 differed only slightly from S1 and S3, but differed greatly against the second group of samples. And as anticipated, there was a bigger difference in performance between the two types of models (numbers 1,2,3 with a hole vs. numbers 4,5,6 without a hole) (Figures 8-10) which was confirmed by DIC strain mapping.

The T2 test series was conducted with much greater impact energy $(130 \mathrm{~J})$ in order to produce a controlled fracture. The impact energy, velocity decay and force decay were similar in models S3 and S4 (Figures 9, 10a,b) and the fracture patterns were nearly identical in location, size and propagation characteristics (Figure 7). This can be characterized as a closed linear vault fracture, and is very similar in nature to the fracture expected on a real human skull after an impact of this kind. ${ }^{22}$ Further analysis of the fracture based on high-speed photography revealed that the origin of the crack was the thinnest part of the skull (lower nuchal lines) and propagation then occurred towards the point of impact, from below the occipital condyle area and propagating up to the inferior nuchal line all the way through the superior nuchal line and terminating at the occipital bone, agreeing with earlier reports based on the behaviour of real skulls. ${ }^{22}$

Our tested skulls were biofidelic to the extent that they precisely copied the natural structure of the skull bone in shape and form. However, the 3D-printed material is an industrial polymer (ABS) rather than real bone, and the refinement of the printing process of the finest microstructures was in the order of $400 \mu \mathrm{m}$, which inevitably meant that very fine trabeculae in the inner sandwich structure of the skull wall were not reproduced. However, this also meant there was no need to take into account biological variations such as dry versus wet bone properties, because the material in all models was uniform ABS. Other parameters such as fall energy, constraints and contact surfaces were also replicated in the best possible manner. As a striking surface, material similar in mechanical properties to the silicone in the Humanetics Hybrid III headform was used in order to simulate the skin-muscle-hair interface. Because we carried out a comparative study, both the affected and non-affected skull models had the same material properties, these of ABS, thus allowing comparisons to be made primarily driven by the geometric effect of the bone excision. 
Furthermore, the biopsy hole was filled artificially in silico using the ScanIP software, which therefore only approximates the actual shape of a normal skull. The "plug" was of a uniform thickness, but there was still an element of manipulation which might lead to uneven tangential surfaces within the skull vault. Further studies should be conducted to highlight the influence of the void location with respect to the strike location. Indeed, a hole in the thinnest part of the skull might have a much greater effect on the formation and propagation of cracks, and interactions with the location of impact, compared to the models we tested, in which the hole was in one of the thickest parts of the skull. A more complete model, such as a skull attached to the neck, would also provide more realistic responses upon loading. Furthermore, testing with cadaveric skulls would provide validation and also a benchmark for the DIC experimental data, highlighting the potential for future research on this topic.

The present study was an exploration for a 'subject specific' application of 3D printing technology to provide solution in a clinically relevant problem. Examining more patients would allow the methodology to be assessed against biological variability, but in here it is assessed as a complete tool from scan to model. It should then be seen under the prism of some overarching limitations which all have to do with the use of this brand new technology and its application in this context. This goes beyond the specific limitations of how and why present tests were carried out. One such basic concern would be, for instance, the reliability and reproducibility of the 3D printed analogues. This has been addressed in extensive studies of one of the authors ${ }^{12}$ and this rapid prototyping technique produces models which are mechanically performing in quasistatic mode within $5 \%$ of each other in all cases. In testing the qualitative behaviour of the model-skulls used here, which were examined and smashed in a stochastic manner, also showed high reproducibility. The cracks patterns were identical and considering that this was the result in impact and resulted in fracture it should have been quite variable. On the contrary the cracks patterns were the same indicating that both factors (3D reproducibility and bone structure) were replicated to a high degree of repetition. A further question on how similar is the 3D skull to the actual natural skull has not been addressed here in this work which was concerned with aspects of testing and producing reliable physical models. Some simpler and smaller size bone structures, like vertebrae ${ }^{12}$, though, have been replicated though in full and have shown that 3D physical models can be made to resemble the natural counterparts fully and even mechanically ${ }^{12}$.

\section{Conclusions}

A combination of CT scanning, 3D printing, DIC analysis, and drop-tower tests allowed us to study the real-time loading of mechanically compromised skulls. DIC analysis is useful for the measurement of full-field displacements and strains, especially in noncontact mode. Our experiments confirmed that a hole in the occipital region has a direct influence on strain propagation in the skull, but even though the hole increased the stress (evidenced by the concentration and level of strain seen around the hole), the magnitude was not sufficient to trigger a failure initiating from the hole. Instead, the failure was initiated elsewhere in models with and without the lesion. Although the occipital lesion did not influence the initiation or propagation of cracks, it highlighted that the peripheral area of the excision experiences a much greater strain concentration compared to the rest 
of the occipital part of the skull. Recent advances in data collection and 3D printing make it feasible that analogue models, such the one used in this study are possible to construct and may be proven to be instrumental in providing life-changing results in patientspecific cases.

\section{Acknowledgments}

We acknowledge the work, skill and expertise offered by Karl Norris in the mechanical workshop of Cranfield University; Jolyon Cleaves of Vision Research for providing the high-speed cameras. Ethical approval was granted by Cranfield University Research and Ethics committee (CURES). This paper is dedicated to one of the authors, Dr Mike Gibson, whose untimely death is a great loss to us all.

\section{Funding and conflicts of interest}

This work was supported by a Cranfield University internal grant of (CF), a project grant from RCDM Birmingham (PZ), and in-kind support from Vision Research. The authors have no conflicts of interest to declare.

\section{Data accessibility}

The data in this manuscript is available through the Cranfield University CORD data depository and preservation system at https://cranfield.figshare.com.

\section{Authors' agreement and declarations}

All authors contributed to this research and to the preparation of the manuscript.

\section{Authors' contributions}

Conceptualization: CF; Data curation: CF, TL, IH; Formal analysis: CF, TL, GT;

Funding acquisition: CF, PZ; Investigation: CF, TL; Methodology: CF, IH, GT; Project administration: CF, MG, PZ; Resources: CF, IH, PZ; Software: CF; Supervision: MG, GT, PZ; Validation: CF, MG; Visualization: CF; Writing (original draft): CF; Writing (review and editing): CF, GT, PZ.

\section{References}

1. NHS Digital. Hospital Episode Statistics. http://content.digital.nhs.uk/hes (accessed 2 May 2018).

2. Benjamin DJ. The efficacy of surgical treatment of cancer -20 years later. Med Hypoth 2014; 82:412-420.

3. Clark C, Morgan C, Sonstegard D and Matthews L. The effect of biopsy-hole shape and size on bone strength. J Bone Joint Surg 1977; 59:213-217.

4. Errani C, et al. Current concepts in the biopsy of musculoskeletal tumors. Sci World $J$ 2013; 2013:538152.

5. Monea AG, et al. The relation between mechanical impact parameters and most frequent bicycle related head injuries. J Mech Behav Biomed Mater 2013; 33:3-15. 
6. Grassi L et al. Experimental validation of finite element model for proximal composite femur using optical measurements. J Mech Behav Biomed Mater 2013; 21:86-94.

7. Hisley D, Gurganus J and Drysdale A. Experimental methodology using digital image correlation to assess ballistic helmet blunt trauma. J Appl Mech 2010; 78:051022.

8. Jones $\mathrm{M}$ et al. Development and validation of a physical model to investigate the biomechanics of infant head impact. Forens Sci Int 2017; 276:111-119.

9. Sutradhar A et al. Experimental validation of 3D printed patient-specific implants using digital image correlation and finite element analysis. Comp Biol Med 2014; 52:8-17.

10. Palanca M, Tozzi G and Cristofolini L. The use of digital image correlation in the biomechanical area: a review. Int Biomech 2015; 3:1-21.

11. Inglis S. 3D Printing in the NHS and healthcare sciences. IPEM SCOPE 2016; 25:10-13.

12. Franceskides C. Subject specific functional model of hard and soft tissues: Skull and Spine. PhD Thesis, Cranfield Univ, 2018.

13. Appleby-Thomas GJ, Fitzmaurice B, Hameed A, et al. On differences in the equation-of-state for a selection of seven representative mammalian tissue analogue materials, Journal of the Mechanical Behavior of Biomedical Materials, 2018; 77 586-593.

14. Vassolera JM and Fancelloa EA. Error analysis of the digital image correlation method. Asoc Argentina de Mecán Comp 2012; 29:6149-6161.

15. Pan B, Lu Z and Xie H. Mean intensity gradient: An effective global parameter for quality assessment of the speckle patterns used in digital image correlation. Optics Lasers Eng 2010; 48:469-477.

16. Lecompte D et al. Quality assessment of speckle patterns for digital image correlation. Optics Lasers Eng 2006; 44:1132-1145.

17. Sutton MA, Orteu JJ and Schreier H. Image correlation for shape, motion and deformation measurements. New York: Springer, 2009.

18. Office of Crash Worthiness Standards. Parts list and drawings subpart E. Hybrid III $50^{\text {th }}$ percentile male.

https://www.unece.org/fileadmin/DAM/trans/doc/2009/wp29/HIII_50th_Male_Dra wings.pdf, (1997, accessed 2 May 2018).

19. US Army Medical Research Acquisition Activity. Notice for ballistic load sensing headform system. www.fbo.gov/index (2014, accessed 2 May 2018).

20. Cantrell J et al. Experimental characterization of the mechanical properties of 3Dprinted ABS and polycarbonate parts. Adv Optical Meth Exp Mech 2016; 3:89-105.

21. Zou $\mathrm{R}$ et al. Isotropic and anisotropic elasticity and yielding of $3 \mathrm{D}$ printed material. Composites B 2016; 99:506-513.

22. Galloway A. Broken bones, anthropological analysis of blunt force trauma. Springfield, Ilinois: Charles C Thomas, 1999. 
Table 1 - HSCIS data showing the number of falls resulting in head injuries and the number of skull biopsies between 2010 and 2015 .

\begin{tabular}{|l|l|l|}
\hline Year & $\begin{array}{l}\text { Number of falls } \\
\text { resulting in head injury }\end{array}$ & $\begin{array}{l}\text { Number of skull } \\
\text { biopsies }\end{array}$ \\
\hline $2010-2011$ & 229,177 & 421 \\
$2011-2012$ & 226,002 & 450 \\
$2012-2013$ & 211,895 & 467 \\
$2013-2014$ & 214,900 & 406 \\
$2014-2015$ & 217,529 & 461 \\
\hline Total & $1,099,503$ & 2205 \\
\hline
\end{tabular}

Table 2 - Cube compressive directional properties and anisotropic magnitudes.

\begin{tabular}{|c|c|c|}
\hline Specimen & $\begin{array}{c}\text { Gradient } \\
(\mathrm{kN} / \mathrm{mm})\end{array}$ & $\begin{array}{c}\text { Gradient } \\
\%\end{array}$ \\
\hline 90 degrees & 23.3 & 100 \\
0 degrees & 19.7 & 85 \\
\hline $\begin{array}{c}45 \text { degrees } \\
\mathrm{Y}\end{array}$ & 22.2 & 95 \\
$\begin{array}{c}45 \text { degrees } \\
\mathrm{Z}\end{array}$ & 21.2 & 91 \\
\hline
\end{tabular}




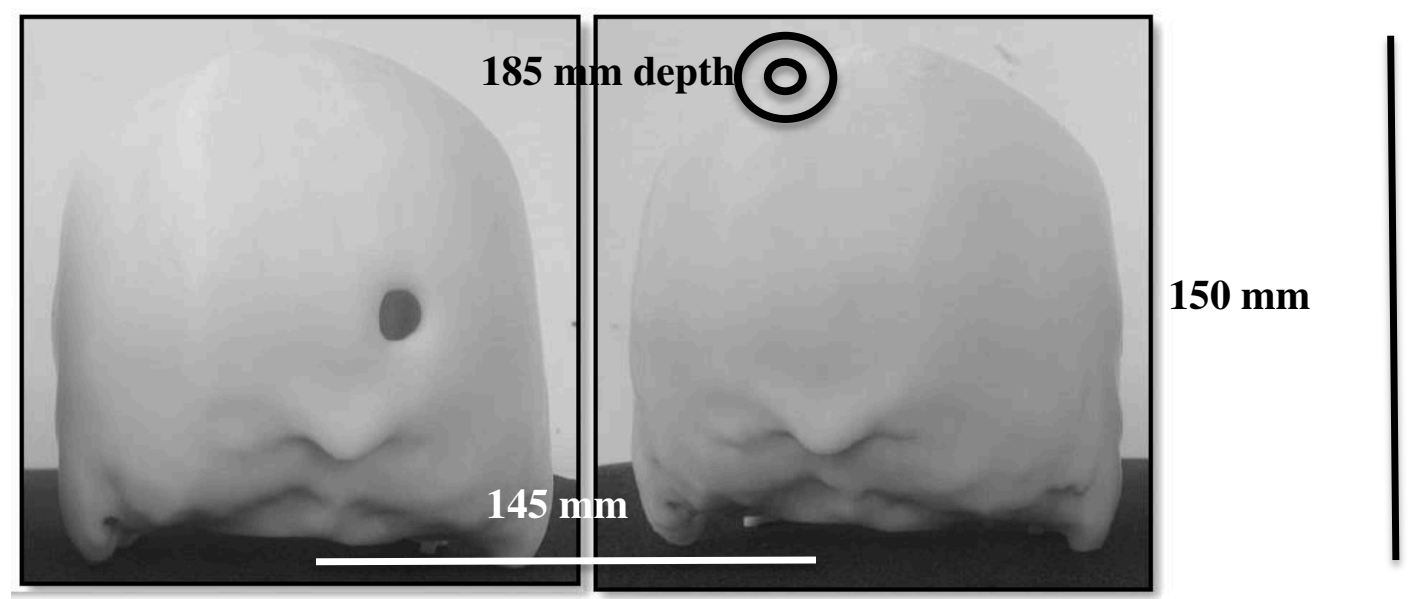

Figure 1 - The two distinct skull geometries, with (left) and without (right) the excision. Depth, Width and Height of the skull are shown by the respective scales $(185,145$, $150 \mathrm{~mm})$.

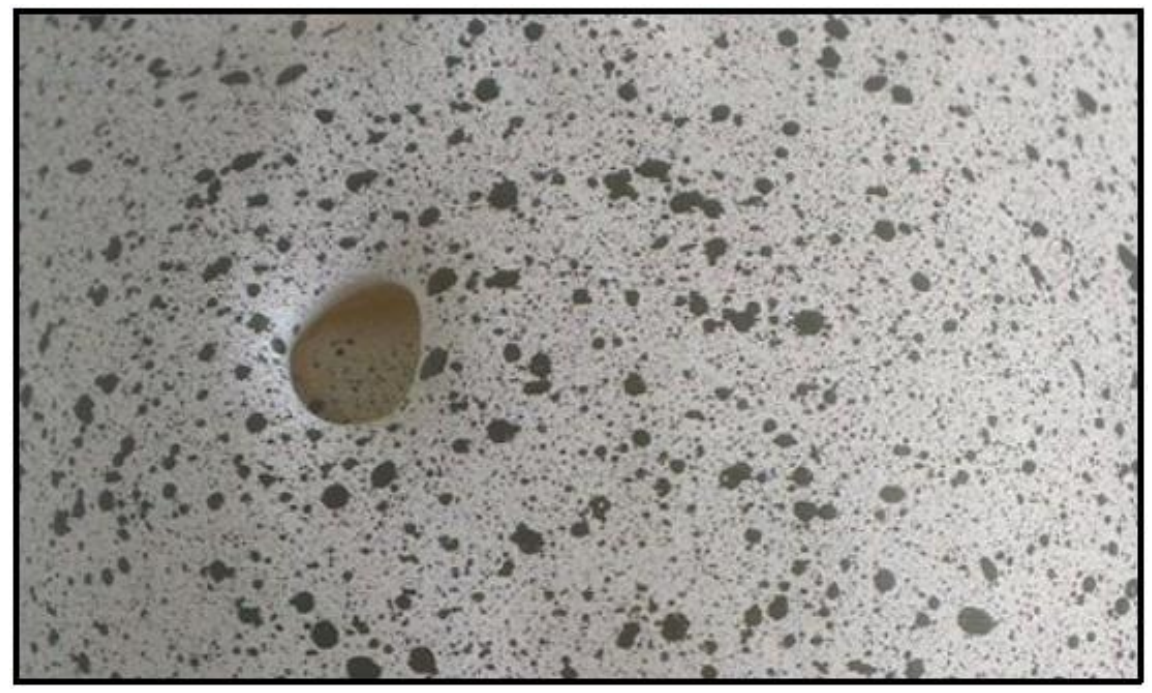

Figure 2 - Typical speckle pattern, exemplified using a model with an excision to show the brain simulant within. 


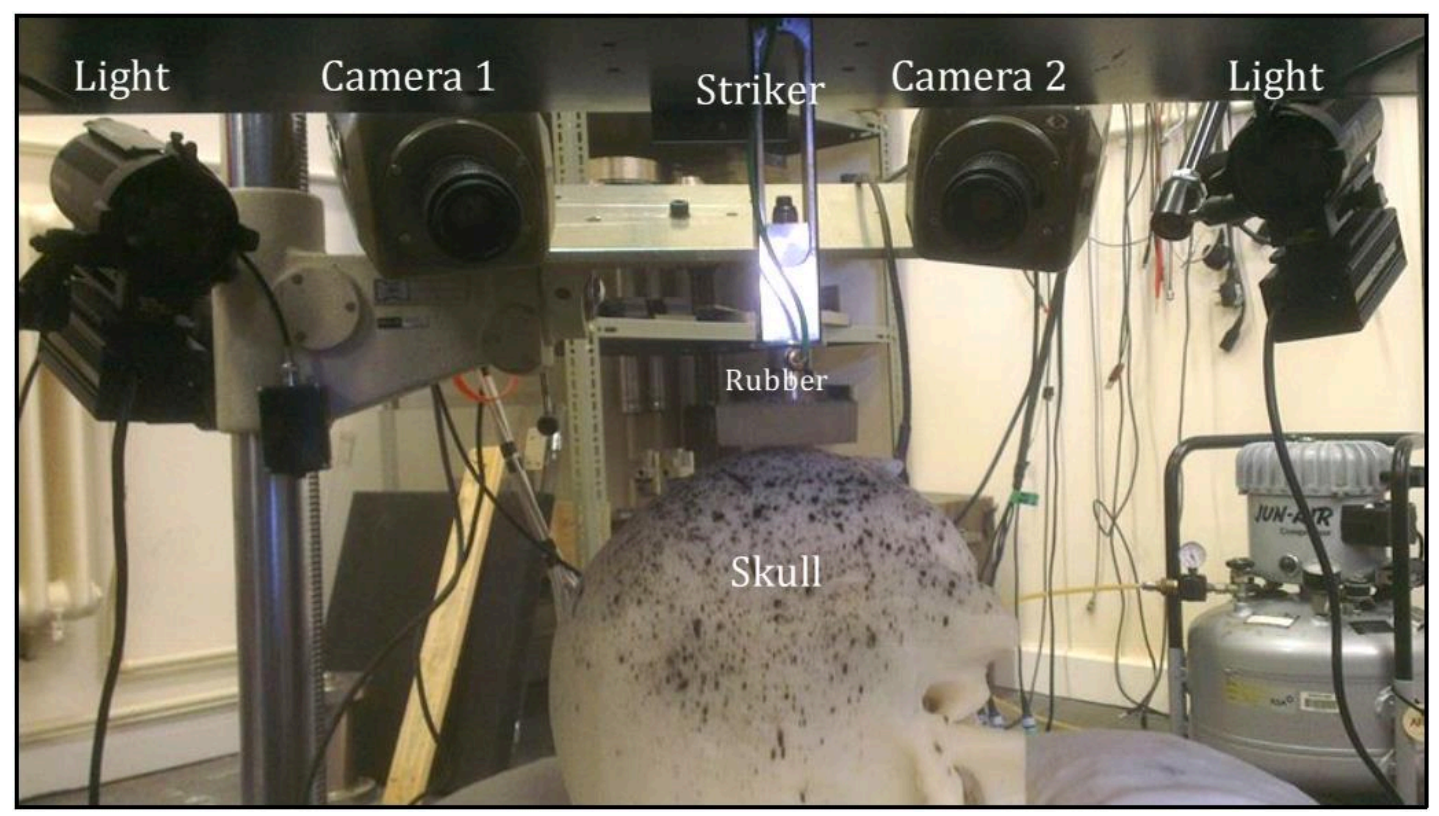

Figure 3 - The experimental setup for the drop test.
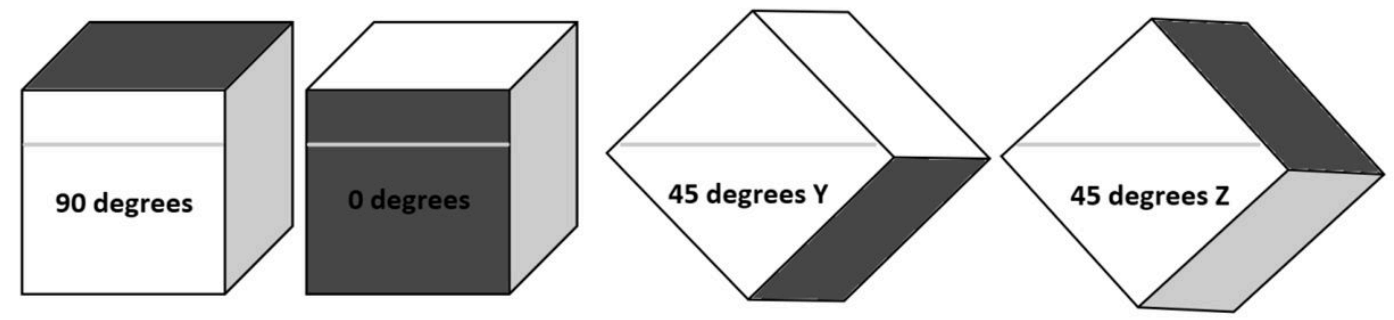

Figure 4 - Direction of load (dark gray surface) and orientation of layer deposition (line) in 3D-printed cubes. 


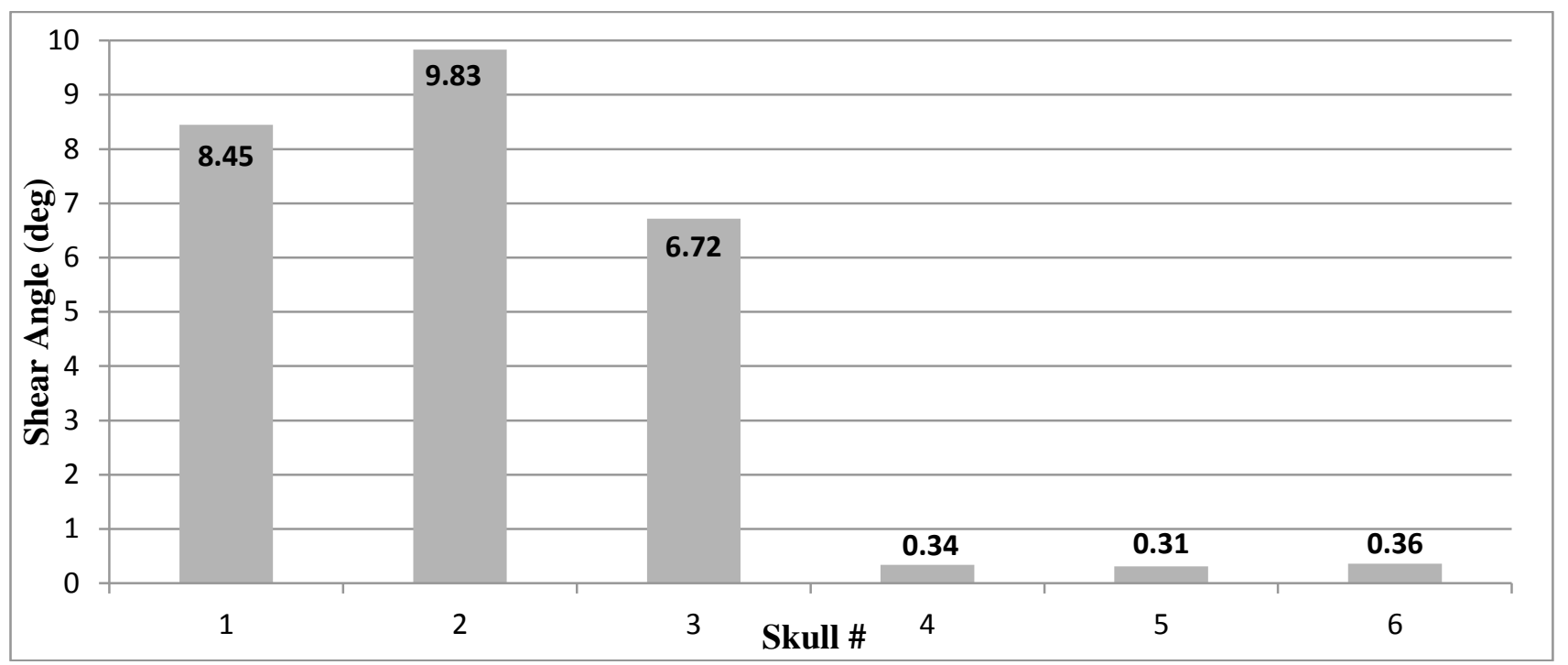

Figure 5 - Shear angle proximal to excision for the six skull models under T1 test conditions. Statistically significant difference ANOVA P=0.001.

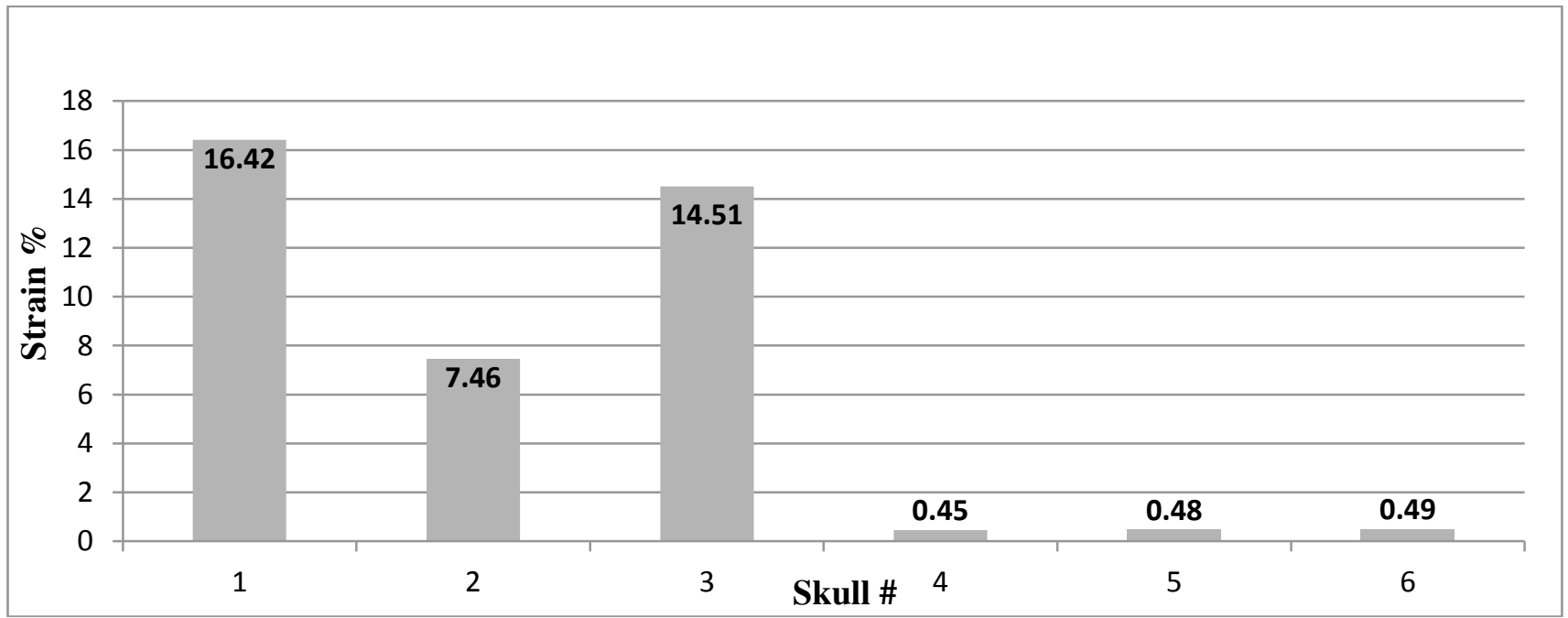

Figure 6 - Absolute maximum principal strain recorded in the excision area for the six skull models under T1 test conditions (for samples S4-S6 there was no excision as such, so this is strain in this same region). Statistically significant difference ANOVA P=0.011. 

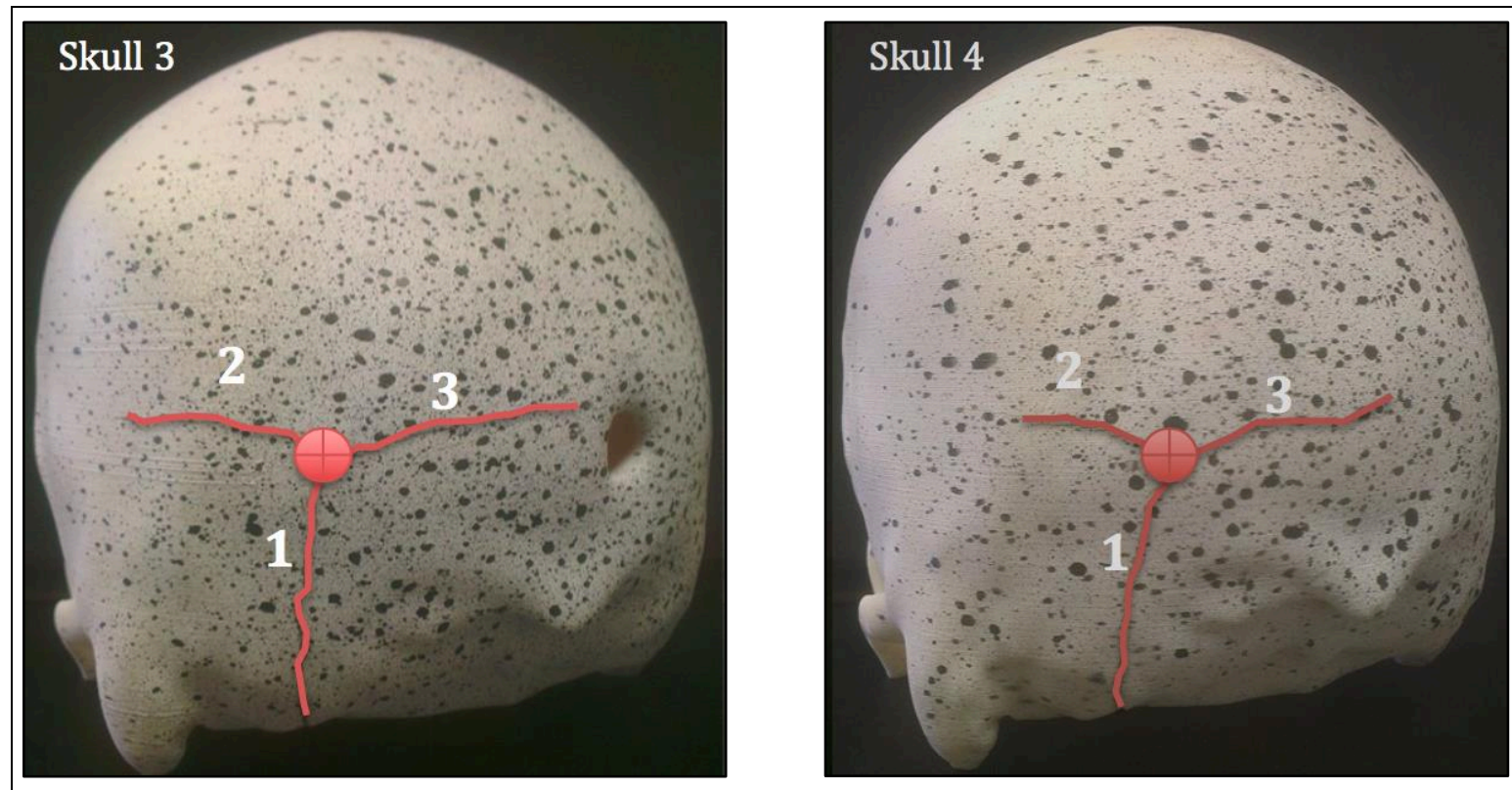

Figure 7 - The initiation, propagation and magnitude of cracks under T2 test conditions in skull models with (left) and without (right) an excision.
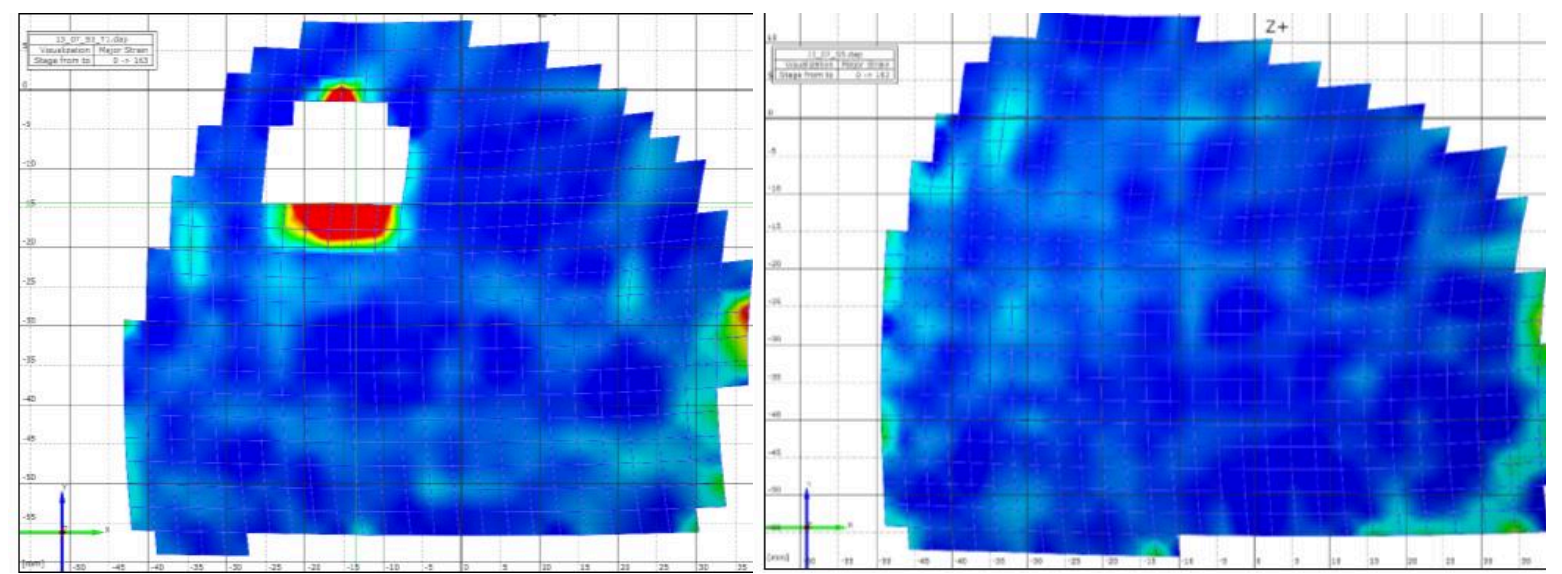

Figure 8 - Screenshots of the Aramis program showing strain concentration around the perimeter of the excision (left) but no equivalent strain concentration in the healthy skull model (right). This view is at $90^{\circ}$ to the one in Figure 7 , hence the point of impact here is at the left-hand side of the frame. 


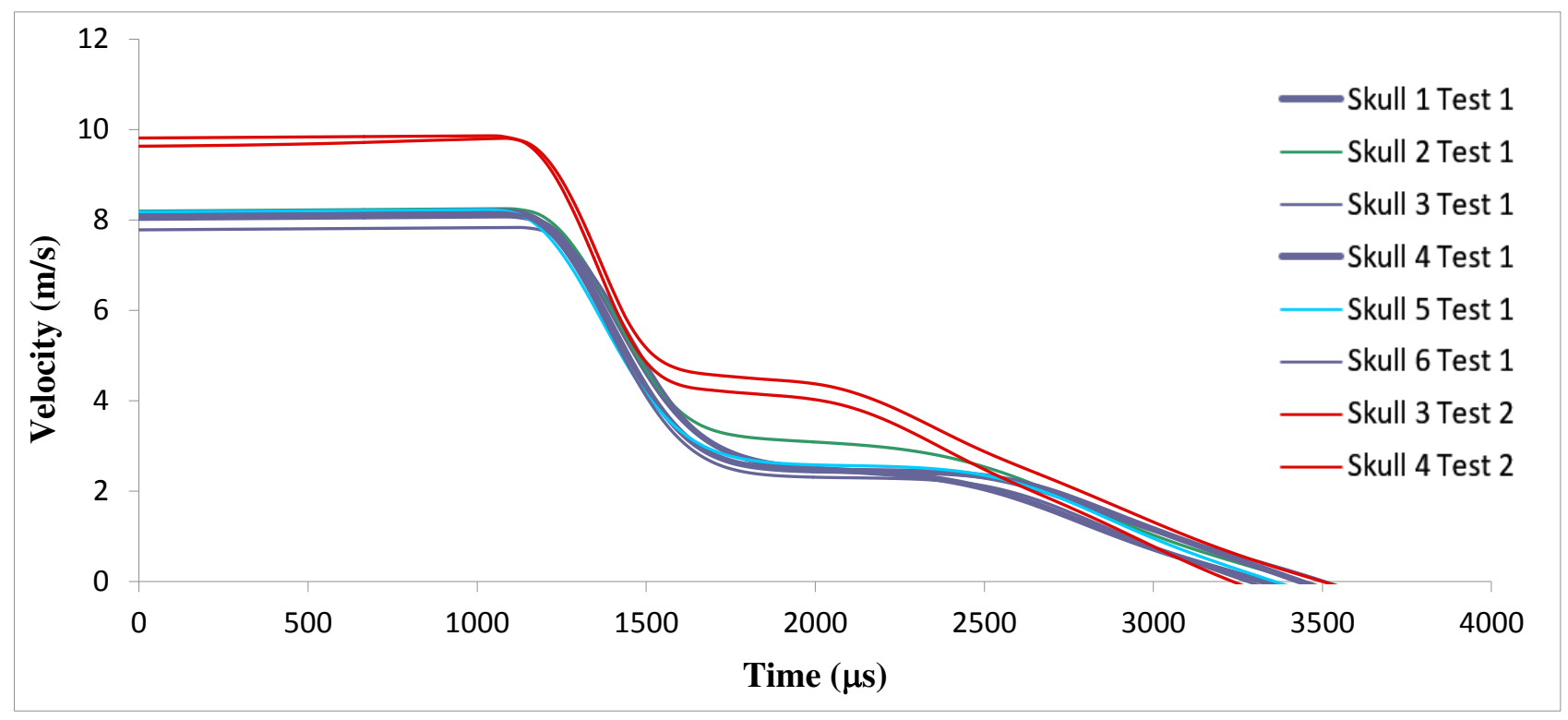

Figure 9 - Impact velocity vs time decay in the T1 and T2 test environments. 


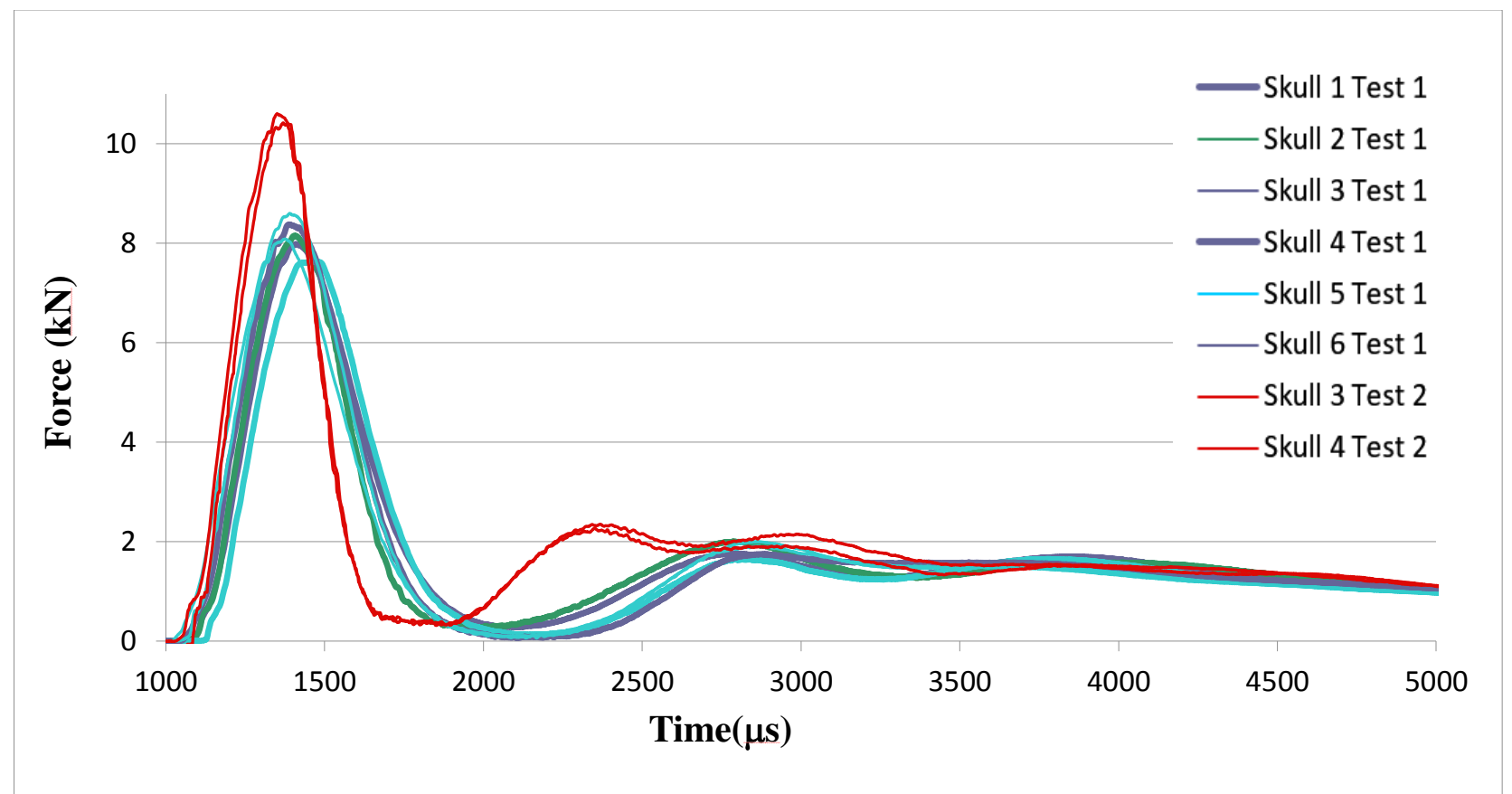

(a)

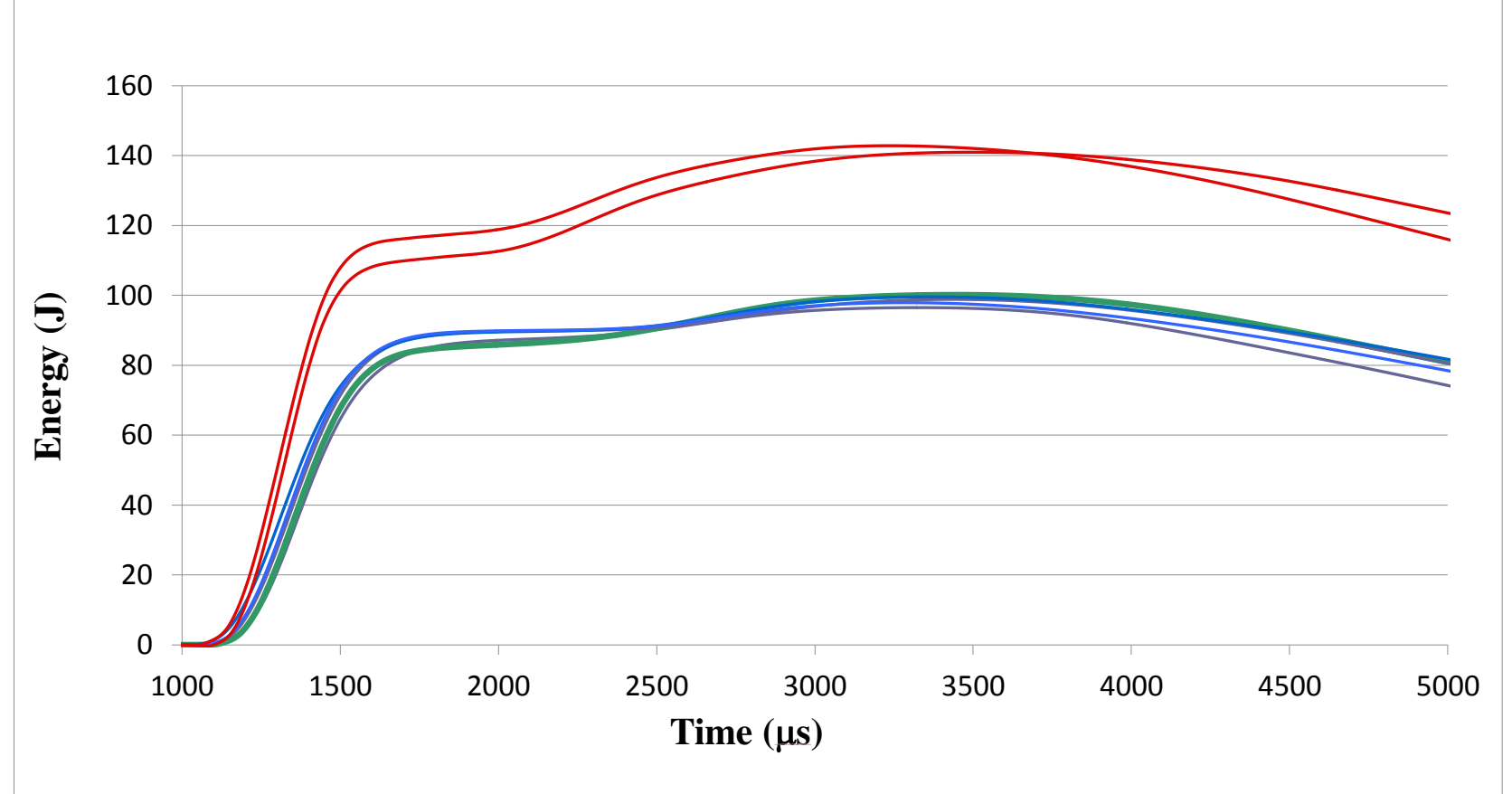

(b)

Figure 10 - (a) Impact force and (b) Impact energy vs time decay for the T1 and T2 test environments. 


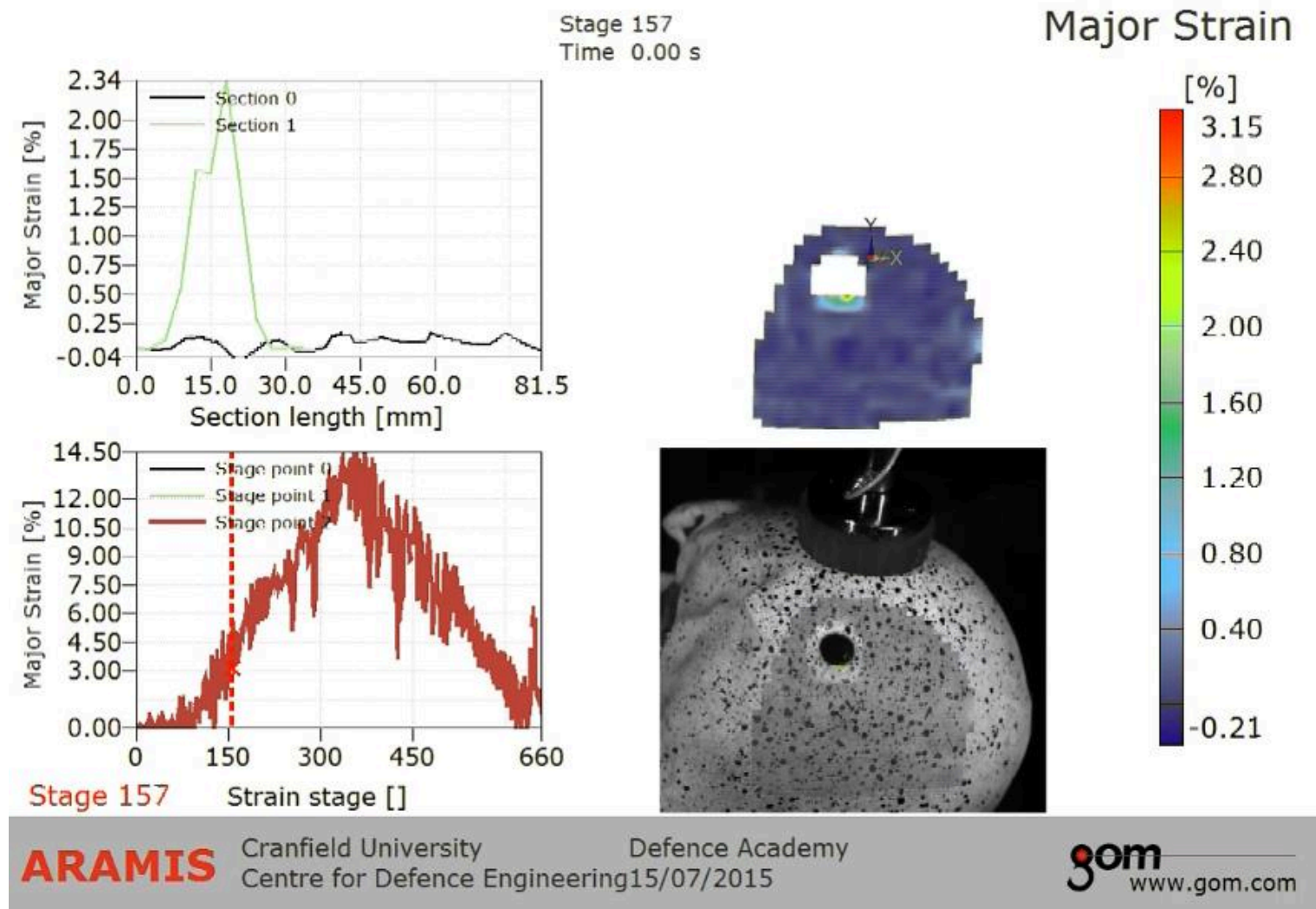

Figure 11 - Test screen in Aramis GOM software displaying in synchronous mode principal strain (near the hole), strain mapping, video high speed play and range of strain all evident in one screen frame. The dashed red line (bottom left screen) shows where exactly in the overall time sequence of events this photo frame is taken and helps interpretations, of the cause/effect kind, to be made. 